\title{
A Method to Identify the Nonlinear Stiffness Characteristics of an Elastic Continuum Mechanism
}

\author{
Bastian Deutschmann ${ }^{1}$, Tong Liu $^{1,2}$, Alexander Dietrich ${ }^{1}$, Christian Ott $^{1}$, and Dongheui Lee ${ }^{1,2}$
}

\begin{abstract}
The humanoid robot David is equipped with a novel robotic neck based on an elastic continuum mechanism (ECM). To realize a model-based motion control, the six dimensional stiffness characteristics needs to be known. The paper presents an approach to experimentally identify the stiffness characteristic using a robot manipulator to deflect the ECM and measure the Cartesian wrenches and Cartesian poses with external sensors. A three-step process is proposed to establish Cartesian wrench and pose pairs experimentally. The process consists of a simulation step, to select a good model, a second step that extracts effective poses from workspace which are sampled experimentally and the third step, the pose sampling procedure in which the robot drives the ECM to these effective poses. A full cubic polynomial regression model is adopted based on simulation data to fit the stiffness characteristics. To extract the poses to be sampled in the experiments, two different approaches are evaluated and compared to ensure a well-posed identification. The identification process on the hardware is performed by using Cartesian impedance and inverse kinematics control in combination to comply with the physical constraints imposed by the ECM.
\end{abstract}

Index Terms-Model Learning for Control, Compliant Joint/Mechanism, Soft Material Robotics

\section{INTRODUCTION}

$\mathbf{I}^{\mathrm{N}}$ $\mathrm{N}$ recent years, humanoid robotic systems have been equipped with passively compliant mechanical elements which enables the robot to dynamically interact with the environment. Based on this design principle, the DLR robot David (see Fig. 1) has been developed. In [1], an elastic continuum mechanism (ECM) is introduced which serves as a neck or spine for David. The ECM consists of a silicone structure which is deformed by a rigid platform at the top end at which the tendon actuation is attached. This is a common actuation scheme among such mechanism, e.g. [2], [3], and it enables the neck to rotate about all Cartesian axis, see Fig.1. In [4], a model-based controller is designed based on a simplified model of the system, assuming that the system can be modeled as a rigid-body, the head, on top of a nonlinear Cartesian spring. This nonlinear Cartesian spring should embody the stiffness characteristics of the ECM that can be described by the mapping from a Cartesian pose to a Cartesian wrench. Other control approaches are also feasible

Manuscript received: September, 08, 2017; Revised December, 14, 2017; Accepted January, 14, 2018.

This paper was recommended for publication by Editor Paolo Rocco upon evaluation of the Associate Editor and Reviewers' comments.

1 All Authors are with the Institute of Robotics and Mechatronics of the German Aerospace Center (DLR) bastian. deutschmann@dlr.de

2 Tong Liu and Donghieu Lee are with the Human-centered Assistive Robotics group of the Technical University of Munich (TUM) dhlee@tum.de

Digital Object Identifier (DOI): see top of this page.
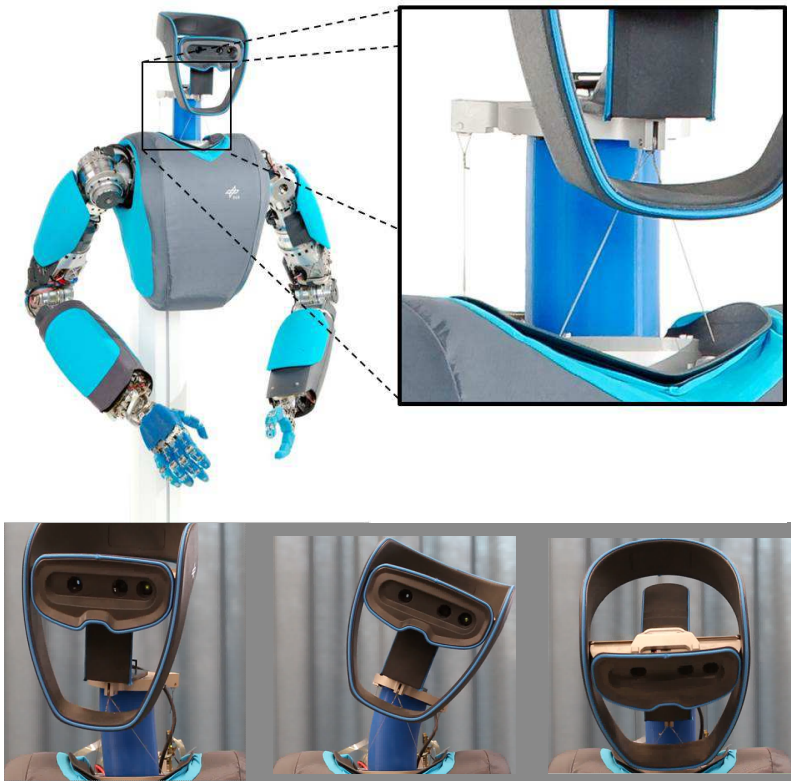

Fig. 1. The neck of the DLR robot David [1] and its motion capabilities

such as linear, robust controllers [5], impedance controllers [6] or model-based observers for online state estimation such as Kalman Filters. All mentioned alternatives desire the nonlinear stiffness characteristics or the linearized stiffness matrix of the ECM. State-of-the-art methods that describe the stiffness characteristic of an ECM are often based on continuum mechanical beam models [7], [8] and are solved using finite differences. This approach tends to consume much computation time, e.g. [8] states $28 \mathrm{~min}$ for $1 \mathrm{~s}$ of simulation. The SOFA framework [9] uses the finite element method to simulate the deformation of general soft structures under external loading such as continuum robots and $23 \mathrm{~ms}$ for a computation cycle is reported for a beam-like structure [10]. Another possibility is to apply reduced models that approximate the behavior of the mechanism. For example, the kinematic assumption that the mechanism deforms with constant curvature is used [11], [12]. Recently, model-free techniques are studied to approximate the mapping of a continuum mechanisms between its task space and the associated actuator configuration. This mapping is identified based on experiments in which the mechanism is driven to several positions in the workspace by the actuation system. Positions in the actuation space and the associated workspace are recorded based on which the mapping is trained. By using a neural network for the mapping, [13] proved to be computationally efficient compared to a model from nonlinear beam theory and [14] extended the neural network 


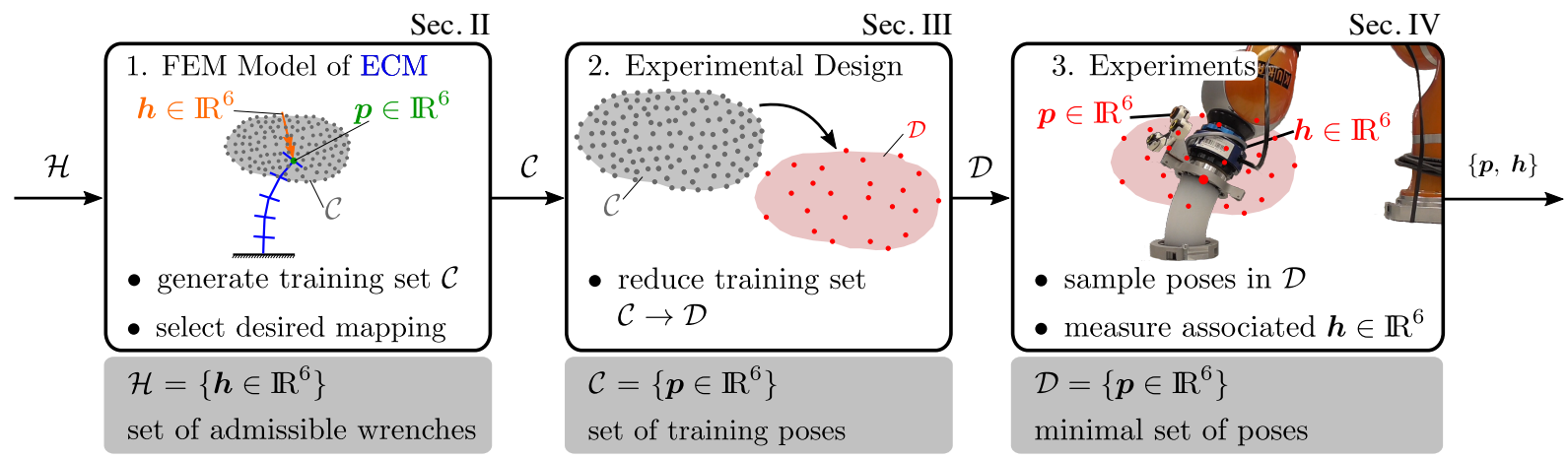

Fig. 2. Overview of the three subsequent steps incorporated in the proposed method to identify the stiffness characteristic. Step 1. corresponds to Sec. II, step 2. corresponds to Sec. III and step 3. corresponds to Sec. IV.

approach to cover possible redundancies. Another modelfree technique is task-parametrized Gaussian mixture models which was successfully applied by [15] in the same context. The main drawbacks that hinder the discussed methods to be used in the present application are as follows: On the one hand, the control cycle of the hardware is $1 \mathrm{kHz}$ [1]. Thus the computational costs of continuum mechanical models are to high. An improvement is expected when using the SOFA framework [9], however a Dirichlet boundary value problem would be necessary to embody the stiffness characteristics, i.e. the mapping from Cartesian pose to Cartesian wrench, which will increase the computational demands even more. On the other hand, the proposed experimental procedures to identify the model-free approaches incorporate only reachable configurations of the actuated mechanism. This yields that the model is not valid in cases of external disturbances.

In this work, we want to establish a model-free approach for the class of continuum mechanisms, that are actuated by a rigid platform whilst no distributed loading along the mechanism is introduced. The mapping should take the Cartesian pose $\boldsymbol{p} \in \mathbb{R}^{6}$ of the rigid platform as an input to compute the associated Cartesian wrench $\boldsymbol{h} \in \mathbb{R}^{6}$ of the ECM resisting this deformation. The main contribution of this paper is to present a novel method to experimentally identify aforementioned mapping. The basic idea is to move the ECM tip pose to predefined static poses. At each pose, an external camera system measures this pose whereas a force torque sensor measures the wrench at the tip. The essential difference to previous works [13]-[15] is that these poses cover more than reachable space of the actuation system to incorporate also poses induced by additional external disturbances. The threestep process of Fig. 2 is proposed, which consists of

1) a simulation to generate a large set of training poses $\mathcal{C}$ which allows to find a suitable mapping from $\boldsymbol{p}$ to $\boldsymbol{h}$,

2) an experimental design procedure to select a minimum set of informative poses $\mathcal{D}$ from the large set $\mathcal{C}$,

3) and an efficient implementation of the experimental procedure to consecutively sample the poses of $\mathcal{D}$ whilst unwanted configuration for the ECM are avoided. As no analytical model of the workspace of the ECM is available, the transitions between subsequent poses cannot be predicted online.

In the third step, a robotic manipulator is connected to the tip of the mechanism similar to [14] to enable an automatic execution of the experimental procedure. However as the method relies on external sensors for pose and wrench sensing, any $6 \mathrm{DoF}$ positioning device would also be feasible. As an example, a polynomial mapping will be employed in the following as it increases the computational efficiency which would be an advantage against e.g. models from nonlinear beam theory. Furthermore, the stiffness matrix $\boldsymbol{K}(\boldsymbol{p}) \in \mathbb{R}^{6 \times 6}$ can be derived analytically which is very useful to study the stiffness behavior of the mechanism in the workspace or for interaction control tasks where a specific stiffness is required.

\section{Simulation}

In this work, a nonlinear mapping from $\boldsymbol{p}$ to $\boldsymbol{h}$ shall be identified based on an experimental procedure. The 6 components of $\boldsymbol{h}=\left(h_{1}, h_{2}, h_{3}, h_{4}, h_{5}, h_{6}\right)^{T} \in \mathbb{R}^{6}$ are $\left(f_{x}, f_{y}, f_{z}, \tau_{x}, \tau_{y}, \tau_{z}\right)$ and the 6 components of $\boldsymbol{p}$ are $\left(x, y, z, \theta_{x}, \theta_{y}, \theta_{z}\right)$. The pose $\boldsymbol{p}$ describes the position and orientation of the top frame respecting the reference frame in the base of the mechanism. The wrench is exerted at the top frame respecting the reference frame in the base. The location of the respective frames is illustrated in Fig. 3.

The simulation covers two fundamental steps for the proposed method as illustrated in Fig. 2. First, a training data set $\mathcal{C}$ of wrench-pose pairs is generated to cover the desired space of the mapping. Second, a model is selected and trained to evaluate the fitting accuracy which is also the basis of the model-based experimental design in section III-A. A full cubic-polynomial model is applied in this work although other mappings, such as a neural network or a Gaussian process regression, are applicable as well.

\section{A. Generating the Training Set}

A set of 1700 admissible wrenches $\mathcal{H}=\left\{\boldsymbol{h} \in \mathbb{R}^{6}\right\}$ is randomly generated to cover well the reachable workspace of the ECM and compute the respective pose, summarized in the training set $\mathcal{C}$. To simulate the deformation of the ECM, a static FEM model based on [16] is utilized which takes a Cartesian wrench as input. The generated set $\mathcal{C}$ is used to train and select the full cubic polynomial model. 


\section{B. Polynomial Model}

Linear, quadratic and cubic relationships between $p$ and each wrench component $h_{j}$ for $j=1 \ldots 6$ are studied using the formula

$$
h_{j}^{i}=\left(\boldsymbol{x}^{i}\right)^{T} \boldsymbol{\beta}_{j}+\epsilon_{j}^{i},
$$

where $h_{j}^{i}$ denotes the $i$-th observation of $h_{j}, \boldsymbol{\beta}_{j}$ is a vector containing all polynomial coefficients to be estimated for the $j$-th wrench component, $\epsilon_{j}^{i}$ denotes the $i$-th observation error of the $j$-th wrench component and $\left(\boldsymbol{x}^{i}\right)^{T} \in \mathbb{R}^{k}$ denotes the regressor-row containing the information of the $i$-th pose observation. Depending on the polynomial order, $\boldsymbol{x}^{i}$ takes different forms.

Supposing $\boldsymbol{p}^{i}=\left(x^{i}, y^{i}, z^{i}, \theta_{x}^{i}, \theta_{y}^{i}, \theta_{z}^{i}\right)^{T}$ denotes the $i$-th observation of pose, for the linear case we have

$$
\boldsymbol{x}^{i}=\left(\begin{array}{lllllll}
1 & x^{i} & y^{i} & z^{i} & \theta_{x}^{i} & \theta_{y}^{i} & \theta_{z}^{i}
\end{array}\right)^{T} \in \mathbb{R}^{7},
$$

for the quadratic case we have

$$
\begin{aligned}
& \boldsymbol{x}^{i}=\left(\begin{array}{lllllllll}
1 & x^{i} & y^{i} & \cdots & \theta_{z}^{i} & \left(x^{i}\right)^{2} & x^{i} y^{i} & \cdots & x^{i} \theta_{z}^{i}
\end{array}\right. \\
& \left.\cdots\left(y^{i}\right)^{2} \quad y^{i} z^{i} \quad \cdots \quad\left(\theta_{z}^{i}\right)^{2}\right)^{T} \in \mathbb{R}^{28},
\end{aligned}
$$

and for the cubic case we have

$$
\begin{aligned}
& \boldsymbol{x}^{i}=\left(\begin{array}{lllllll}
1 & x^{i} & \cdots & \psi^{i} & \left(x^{i}\right)^{2} & x^{i} y^{i} & \cdots
\end{array}\left(\theta_{z}^{i}\right)^{2}\right. \\
& \left.\cdots\left(x^{i}\right)^{3} \quad\left(x^{i}\right)^{2} y^{i} \quad \cdots \quad x^{i} y^{i} z^{i} \quad \cdots \quad\left(\theta_{z}^{i}\right)^{3}\right)^{T} \in \mathbb{R}^{84} .
\end{aligned}
$$

In general, the regressor row $\left(\boldsymbol{x}^{i}\right)^{T}$ has dimension $k=\left(\begin{array}{c}d+n \\ d\end{array}\right) \in \mathbb{R}$ where $n$ (=6 in this case) is the number of independent variables and $d \in \mathbb{R}$ is the polynomial degree. Stacking $N>k \in \mathbb{R}$ observations of $h_{j}$ into vector form yields

$$
\underbrace{\left(\begin{array}{c}
h_{j}^{1} \\
h_{j}^{2} \\
\vdots \\
h_{j}^{N}
\end{array}\right)}_{\boldsymbol{h}_{j}}=\underbrace{\left(\begin{array}{c}
\left(\boldsymbol{x}^{1}\right)^{T} \\
\left(\boldsymbol{x}^{2}\right)^{T} \\
\vdots \\
\left(\boldsymbol{x}^{N}\right)^{T}
\end{array}\right)}_{\boldsymbol{X}} \boldsymbol{\beta}_{j}+\underbrace{\left(\begin{array}{c}
\epsilon_{j}^{1} \\
\epsilon_{j}^{2} \\
\vdots \\
\epsilon_{j}^{N}
\end{array}\right)}_{\boldsymbol{\epsilon}_{j}}
$$

where $\boldsymbol{X} \in \mathbb{R}^{N \times k}$ is the regressor matrix and $\boldsymbol{\epsilon}_{j} \in \mathbb{R}^{N}$ is the vector of residuals for the wrench component $j$. Equation (5) represents a general regression problem and the coefficients $\boldsymbol{\beta}_{j}$ can be computed directly.

In the present case, the polynomial regression suffers from a near collinearity problem, meaning that $\left(\boldsymbol{x}^{i}\right)^{T}$ are almost linearly dependent and therefore $\boldsymbol{X}$ is badly conditioned. To judge the presence of near collinearity, the condition number $\kappa(\boldsymbol{X}) \in \mathbb{R}$ of the regressor [17] is computed to $\kappa(\boldsymbol{X})>5 \times 10^{6}$, which is problematic. Possible solutions to overcome the problem of collinearity are utilize normalization approaches [18] or orthogonal polynomials [19]. Furthermore, the concept of "Ridge Regression" [20], or the "Principal Component Regression" (PCR) [21] could be applied. The PCR together with the normalization approach proves to be the most effective reduction technique in the present case to reduce $\kappa(\boldsymbol{X})$ essentially. Within the normalization, the data is restricted to $[-1,1]$. As the PCR utilizes a threshold $b \in \mathbb{R}$ to decide the presence of collinearity, different $b$ will be evaluated in the next paragraph.
TABLE I

RANGE OF EACH WRENCH COMPONENT

\begin{tabular}{c|ccc}
\hline$[\mathrm{N}]$ & $f_{x}$ & $f_{y}$ & $f_{z}$ \\
\hline $\max$ & 30 & 100 & 100 \\
$\min$ & -110 & -100 & -100 \\
\hline \hline$[\mathrm{Nm}]$ & $\tau_{x}$ & $\tau_{y}$ & $\tau_{z}$ \\
\hline $\max$ & 5 & 5 & 5 \\
$\min$ & -5 & -5 & -5 \\
\hline
\end{tabular}

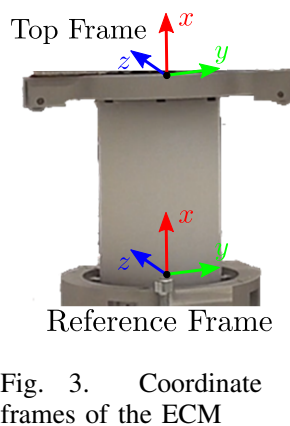

\section{Fitting Results}

The set of admissible wrenches, i.e. the input of the FEM simulation, are defined as the region

$$
\mathcal{H}=\left\{\boldsymbol{h} \in \mathbb{R}^{6} \mid \boldsymbol{h}_{\text {min }}<\boldsymbol{h}<\boldsymbol{h}_{\max }\right\}
$$

The components of $\boldsymbol{h}_{\max }$ and $\boldsymbol{h}_{\min }$ are given in Table I. The maximum and minimum values are chosen to ensure that the training set $\mathcal{C}$ covers well the reachable workspace of the ECM

To evaluate the accuracy of the polynomial model, 300 data pairs from $\mathcal{C}$ are randomly chosen to serve as the test set. The rest is utilized to compute the coefficients $\boldsymbol{\beta}_{j}$. For comparison, the relative error of the wrench component $h_{j}$ is defined

$$
e_{\text {rel }}=\frac{\hat{h}_{j}-h_{j}}{h_{j}},
$$

where $\hat{h}_{j}$ and $h_{j}$ denote the estimated and the observed value of the polynomial model.

The 1-degree polynomial model (linear regression) and the 2-degree polynomial display large prediction errors whereas the 3-degree polynomial model provide a fit with a very low root mean square error (RMSE), see Table II. The 3-degree polynomial ordinary regression has a condition number of , which can be further reduced by using PCR. In the lower part of Table II, three different thresholds $b$ are compared regarding their effect on $\kappa(\boldsymbol{X})$ and the prediction accuracy. An ordinary least square estimation is represented by $b=0$. As $b$ increases, the prediction error grows but the regression becomes better conditioned.

The relative error distribution of each $h_{j}$ by using PCR (with threshold 0.1) onto a 3-degree polynomial regression model is depicted in Fig. 4. The vertical axis represents the amount of prediction points referred to a prediction error level. Most of the predicted wrench components have a relative error less than 0.1. The points with relative error beyond the interval [$0.2,0.2]$ are not displayed as they are located in a small range around zero (from $-2 \mathrm{~N}$ to $2 \mathrm{~N}$ for forces and from $-0.2 \mathrm{Nm}$ to $0.2 \mathrm{Nm}$ for torques) and the absolute deviation of both is also in an acceptable range (smaller than $1 \mathrm{~N}$ for the force prediction and $0.05 \mathrm{Nm}$ for the torque prediction).

\section{EXPERIMENTAL DESIGN}

The design of experiments (DoE) is a technique to extract the important information from a set of collected data [22]. If a model of the process that relates the collected data is known, a model-based design can be set up and the important 
TABLE II

COMPARING THE PREDICTION ERROR OF DIFFERENT POLYNOMIAL DEGREES \& PCR WITH DIFFERENT THRESHOLDS

\begin{tabular}{c|cccccc|c}
\hline \multirow{2}{*}{ degree $d$} & \multicolumn{3}{|c|}{ RMSE [N] } & \multicolumn{3}{c|}{ RMSE [Nm] } & \multirow{2}{*}{$\kappa(\boldsymbol{X})$} \\
\cline { 2 - 7 } & $f_{x}$ & $f_{y}$ & $f_{z}$ & $\tau_{x}$ & $\tau_{y}$ & $\tau_{z}$ & $\kappa .0$ \\
2 & 22.7 & 5.1 & 5.5 & 0.4 & 0.2 & 0.2 & 18.0 \\
3 & 0.9 & 4.5 & 4.6 & 0.0 & 0.1 & 0.1 & 426.6 \\
\hline threshold $b(\mathrm{~d}=3)$ & 0.4 & 0.1 & 0.1 & 0.0 & 0.0 & 0.0 & 9325 \\
\hline 0.0 & $f_{x}$ & $f_{y}$ & $f_{z}$ & $\tau_{x}$ & $\tau_{y}$ & $\tau_{z}$ & $\kappa(\boldsymbol{X})$ \\
0.1 & 0.4 & 0.1 & 0.1 & 0.0 & 0.0 & 0.0 & 9325 \\
0.5 & 0.4 & 0.2 & 0.2 & 0.0 & 0.0 & 0.0 & 464 \\
& 0.7 & 0.9 & 0.9 & 0.0 & 0.1 & 0.1 & 105 \\
\hline
\end{tabular}

information are selected based on some optimality criteria of the information matrix [23]. For the case that no model is given for the process, a model-free design can be used which selects data pairs that span the experimental region as widely as possible with a uniform coverage. The present work studies a polynomial regression problem. Thus, a model-based and a model-free design method in the context of regression is studied in the following.

\section{A. Model-based Design}

Different model-based design criteria are discussed in [22]. In this paper, the commonly used D-optimality is applied which is defined as follows ${ }^{1}$ :

$$
\max _{\boldsymbol{X}} \operatorname{det} \sum_{i=1}^{n} \boldsymbol{x}^{i}\left(\boldsymbol{x}^{i}\right)^{T}=\max _{\boldsymbol{X}} \operatorname{det} \boldsymbol{M}
$$

The matrix $M \in \mathbb{R}^{n \times n}$ is denoted the information matrix [24] and by solving (8), $n \in \mathbb{R}$ regressor rows $\boldsymbol{x}^{i}$ are chosen such that an accurate identification of the parameters can be achieved. In general, $n>k$, which means that the number of observed poses must be larger than the number of parameters. The result of the D-optimal design is a subset $\mathcal{D}$ consisting of $n$ poses out of $\mathcal{C}$. As the experimental region, i.e. the desired workspace of the ECM, cannot be expressed by a continuous function, optimization methods like [25] which provide the optimal design directly, cannot be applied. Therefore, the Fedorov's Exchange Algorithm (EA) [26] is applied to select the $n$ poses that achieve the D-optimality. The basic idea of the EA is to repeatedly search for candidate poses in $\mathcal{C}$ which improves (8) by exchanging this candidate pose with a certain poses in the design set $\mathcal{D}$.

\section{B. Model-free Design}

Here the maximin-distance design is studied [27] that aims to select a subset $\mathcal{D}$ from the training set $\mathcal{C}$ such that the minimum distance between design poses are maximized,

$$
\max _{\mathcal{D}} \min _{\boldsymbol{p}^{1}, \boldsymbol{p}^{2} \in \mathcal{D}} d\left(\boldsymbol{p}^{1}, \boldsymbol{p}^{2}\right)
$$

where $\boldsymbol{p}^{i}$ denotes a certain pose in $\mathcal{D}$, and $d\left(\boldsymbol{p}^{1}, \boldsymbol{p}^{2}\right)=$ $\left\|\boldsymbol{p}^{1}-\boldsymbol{p}^{2}\right\|$ denotes the Euclidean distance between two poses. To solve the maximin-distance optimal design problem, an algorithm is utilized which is similar with the EA: The basic

\footnotetext{
${ }^{1}$ Here, the D-optimality criterion is simplified according to [22]
}
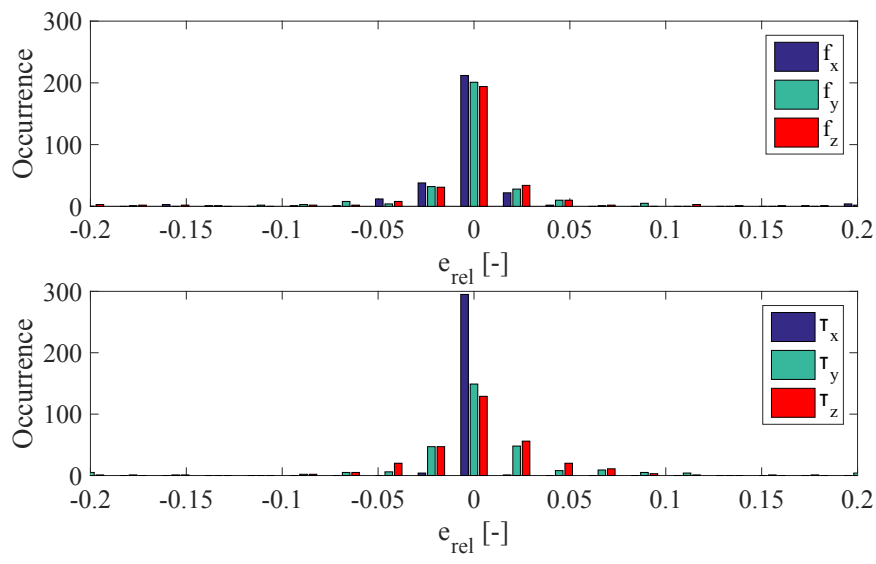

Fig. 4. Histogram of $e_{\text {rel }}$ computed with 300 testing points for each wrench component using PCR with a threshold of $b=0.1$.
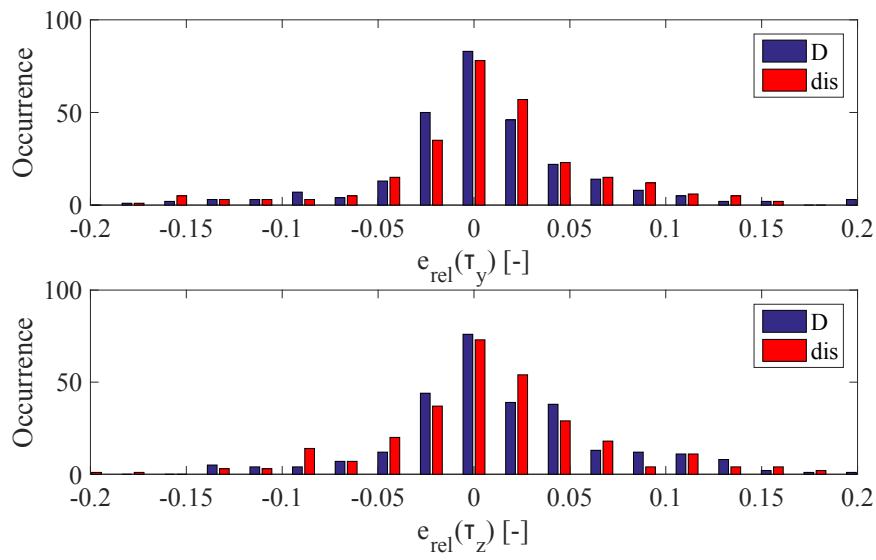

Fig. 5. Histogram of $e_{\text {rel }}$ computed with 200 testing poses of the errorprone wrench components (left: $\tau_{y}$, right: $\tau_{z}$ ) using the data selected by the D-optimal design and the max-min distance.

idea is to randomly choose $n$ poses as the initial design set $\mathcal{D}$. Then, a pose $\boldsymbol{p}^{*}$ from the remainder of $\mathcal{C}$ is randomly chosen and the algorithms checks, if (9) can be increased by replacing a certain pose $\boldsymbol{p}^{i}$ in $\mathcal{D}$ with $\boldsymbol{p}^{*}$. For details see [28].

\section{Performance of the Design Algorithms}

For the DoE, $n=200$ poses have been selected. To compactly compare the results for this desired number of poses, the relative errors for $\tau_{y}$ and $\tau_{z}$ are depicted as they exhibit the highest model errors in the former simulation, see Fig. 4. The fitting results are displayed in Fig. 5 using Doptimal design and the maximin-distance criterion over 300 random poses from the training set $\mathcal{C}$. Most of the testing points feature a relative error within the interval $[-0.2,0.2]$. The other points are located in a small interval around 0 (from $-2 \mathrm{~N}$ to $2 \mathrm{~N}$ for the force prediction and from $-0.1 \mathrm{Nm}$ to $0.1 \mathrm{Nm}$ for the torque prediction) similar to Sec. II-C.

In conclusion, two approaches for the DoE are introduced and compared. The model accuracy resulting in the simulation for both approaches is equally good with a little advantage of the D-optimality which justifies their use for the experimental identification. 


\section{EXPERIMENTS}

In this section, the components of the experimental setup will be introduced. Then, the control and the pose-planning strategy of the robot manipulator are discussed. Afterward, the experimental fitting results are presented.

\section{A. Experimental Setup}

A robot manipulator (here: KUKA LWR 4+ with a JR3 force-torque-sensor (FTS) mounted at the end-effector) is connected to the top of the ECM, see Fig. 6. A marker target, which is to be detected by the camera, is fixed between the top of the ECM and the FTS to measure the associated deflection of the ECM.

The FTS is a six-DOF sensor based on foil strain gauges. In our experiment, we chose the version $100 \mathrm{M} 40 \mathrm{~A} 3$, it enables a standard force measurement range from $-200 \mathrm{~N}$ to $200 \mathrm{~N}$ in $y, z$ directions and from $-400 \mathrm{~N}$ to $400 \mathrm{~N}$ in $x$ direction, and torque measurement about all axes from $-20 \mathrm{Nm}$ to $20 \mathrm{Nm}$. To accurately track the target at the ECM, the K-series optical measurement systems [29] is used. The measurement system is equipped with a camera (three lenses), infrared LEDs and Space-Probe, which also has 9 LEDs. The measurement system can accurately localize the LEDs within its workspace with an accuracy of $37 \mu \mathrm{m}$.

\section{B. Practical Issues}

1) Pose Measurement and Calibration: The poses are obtained by measuring the positions of the LEDs on the marker target and transforming them into the ECM reference frame. To eliminate unintended camera movements during the experiments compromise calibrated transforms, a second marker target is rigidly fixed near the ECM. To avoid systematic errors in the pose measurement, it is essential that the constant transformations from the marker targets to the desired ECM top frame and ECM reference frame are carefully calibrated.

2) Wrench Measurement and Calibration: In the simulation, the wrench data refers to the wrench exerted on the ECM top respecting the ECM reference frame, while the wrench data measured in the experiment is the one applied at the origin of the FTS frame. Thus, the measured wrenches need to be transformed using adjoint transformations [30] based on a constant, calibrated transformation.

The FTS shows offsets in each measured direction. These offsets drift during the experiments, which is supposed to be related to the temperature of the sensor, and result in nonconstant biases of the measurement. The timing characteristic of these offsets are studied and a linear compensation law is adopted between the measured offsets before and after each experiment to reduce their influence.

\section{Implementation of the Experimental Procedure}

As described earlier, the implementation of the pose sampling process for the experiment needs to be carefully executed as configurations that may damage the mechanism or impose unwanted behaviors need to be avoided. For the implementation, the following considerations are made:

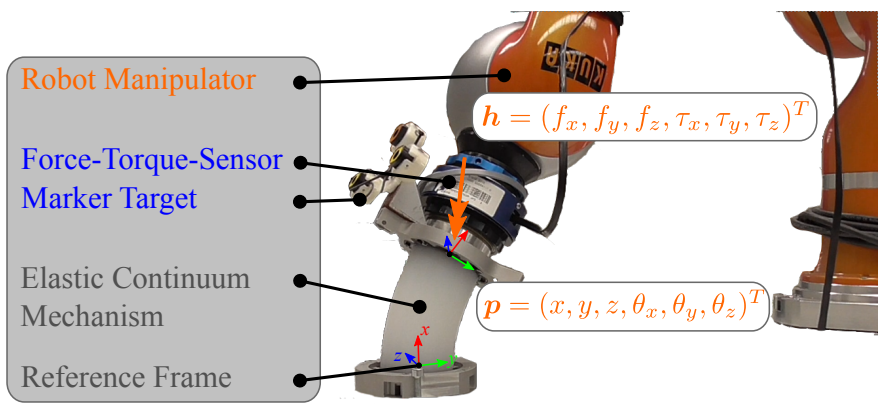

Fig. 6. Experimental setup

1) Comparison of Planning Strategies: One possible planning strategy is to start from the poses close to the initial configuration to those relatively far away from the initial position. After reading the wrench data in one pose the robot moves directly to the next pose. This scheme saves experimental time but it requires knowledge about the path between different configurations. Since the transition between two configurations could cause an unexpected behavior, the path between the poses needs to be designed rigorously.

Another possibility is to return to the initial configuration after each sampling. This scheme is relatively easy to implement and there is no need to concern about the transitions among the poses. Only the transition from the initial configuration to each sample pose needs to be considered. Since the initial configuration is a pose without any deflection, the transition from it to each sample pose is easier to handle than the one among two poses.

2) Comparison of Control Strategies: One strategy is to plan a Cartesian trajectory from the initial pose to the desired pose. The joint space trajectory is calculated the robot is controlled in joint position control mode. In this case each sampled pose requires a specifically designed smooth trajectory such that the ECM can reach the desired pose without unexpected behavior.

Since deflecting the ECM is an interaction between the robot manipulator and the environment, the impedance control seems to be appropriate in this case. The robot is firstly controlled in Cartesian impedance control mode [31] and deflects the ECM towards the desired pose. Due to the controlled compliance, the resulting pose of the ECM differs from the desired pose. After the ECM is stabilized near the desired pose, the control mode therefore switches to joint position control [31], to drive the ECM from the stabilized pose to the desired pose to ensure that the all poses from $\mathcal{D}$ are reached. Since the ECM is already close to the desired pose at that moment, an unexpected motion of ECM can be excluded during the position control mode. The different trajectories in impedance control and joint position control are depicted in the right of Fig. 7.

Based on the above considerations, the experimental procedure can be described by Fig. 7 and the video attached to the present paper. After a pose to be sampled of $\mathcal{D}$ has been selected, the robot drives the ECM from its initial, straight pose in the impedance control mode towards this pose along 

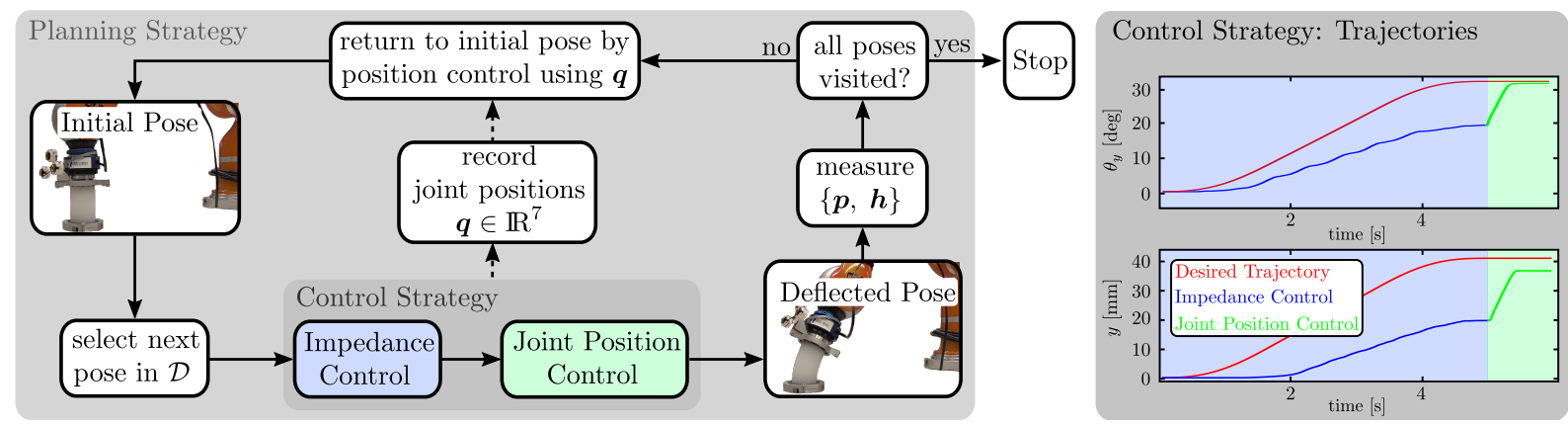

Fig. 7. Block diagram of the implemented procedure to illustrate the subsequent steps during the experiment which can be seen in the attached video also. Right: Example of a measured pose trajectory for the position $y$ and the angle $\theta_{y}$ to examine the different stages while reaching a desired pose.

the planned Cartesian trajectory ${ }^{2}$. After the termination of the Cartesian trajectory interpolator, the inverse kinematics is solved from the current pose to the goal and the robot is driven in joint position controlled mode to reach the desired pose. During the movement from initial pose to the goal, the joint positions of each time instance are recorded and saved in a stack. After reaching the goal the measurements of the pose and the wrench are logged and then the robot returns to the initial pose in joint position control mode in which the desired trajectory are the joint positions saved in the stack. This sequence will be repeated until every pose in $\mathcal{D}$ has been visited. To eliminate the effect of measurement noise, the logging of measurements lasts for $200 \mathrm{~ms}$ and the mean values of pose and wrench are taken.

\section{Experimental Results}

In the experiments, the minimum data sets $\mathcal{D}$ obtained using the D-optimal design and the maximin design (see III-A, III-B) (see III-C) were sampled. To evaluate the fitting results of each data set, one of them is chosen as the training set to identify the polynomial coefficients whereas the other one serves as the testing set to compute the relative errors, and vice versa.

1) Fitting Results using D-optimal Design: Fig. 8 exhibits the histogram of $e_{r e l}$ (7) within the interval $[-0.5,0.5]$. The relative errors of all the torque components and $f_{y}$, $f_{z}$ are mainly distributed in $[-0.1,0.1]$. Compared to these components, $f_{x}$ has a flatter distribution of relative error.

The data points with relative error beyond the interval [$0.5,0.5]$ are plotted in the Actual-vs-Predicted in Fig. 9. They are mainly distributed in $[-5 \mathrm{~N}, 5 \mathrm{~N}]$ for $f_{y}, f_{z}$ and in [$0.4 \mathrm{Nm}, 0.4 \mathrm{Nm}]$ for the torques. Compared to $f_{y}$ and $f_{z}$, $f_{x}$ has a larger absolute prediction error. As the regressor is ill-conditioned $(\kappa(\boldsymbol{X})=12744)$, a PCR is utilized. Table 8 displays the condition number and the RMSE of each wrench component with different thresholds. The threshold $b=0.1$ offers a good trade-off between condition number and prediction performance.

2) Fitting Results using Maximin-distance Design: Fig. 10 displays the histogram of the relative error within the interval $[-0.5,0.5]$. Analogous to the case of the D-optimal design, the relative errors of all the torque components and $f_{y}, f_{z}$ are

\footnotetext{
${ }^{2} \mathrm{~A}$ simple point-to-point motion is concerned and a cubic polynomia timing law is chosen.
}

TABLE III

THE PREDICTION ERROR OF PCR WITH DIFFERENT THRESHOLDS USING EXPERIMENTAL DATA OF BOTH DESIGN METHODS

\begin{tabular}{|c|c|c|c|c|c|c|c|}
\hline \multicolumn{8}{|c|}{ D-optimal design } \\
\hline \multirow[b]{2}{*}{ threshold } & \multicolumn{3}{|c|}{ RMSE [N] } & \multicolumn{3}{|c|}{ RMSE [Nm] } & \multirow[b]{2}{*}{$\kappa(\boldsymbol{X})$} \\
\hline & $f_{x}$ & $f_{y}$ & $f_{z}$ & $\tau_{x}$ & $\tau_{y}$ & $\tau_{z}$ & \\
\hline 0.0 & 1.26 & 0.62 & 0.62 & 0.03 & 0.06 & 0.06 & 12744 \\
\hline 0.1 & 1.26 & 0.89 & 0.72 & 0.03 & 0.06 & 0.07 & 153.26 \\
\hline 0.5 & 3.83 & 3.19 & 3.81 & 0.10 & 0.25 & 0.27 & 25.57 \\
\hline \multicolumn{8}{|c|}{ Maximin-distance design } \\
\hline & \multicolumn{3}{|c|}{ RMSE [N] } & \multicolumn{3}{|c|}{ RMSE $[\mathrm{Nm}]$} & \\
\hline threshold & $f_{x}$ & $f_{y}$ & $f_{z}$ & $\tau_{x}$ & $\tau_{y}$ & $\tau_{z}$ & $\kappa(\boldsymbol{X})$ \\
\hline 0.0 & 1.58 & 0.76 & 0.63 & 0.03 & 0.08 & 0.07 & 21291 \\
\hline 0.1 & 1.53 & 0.99 & 0.85 & 0.03 & 0.08 & 0.01 & 142.67 \\
\hline 0.5 & 3.56 & 3.17 & 5.16 & 0.12 & 0.36 & 0.25 & 29.13 \\
\hline
\end{tabular}
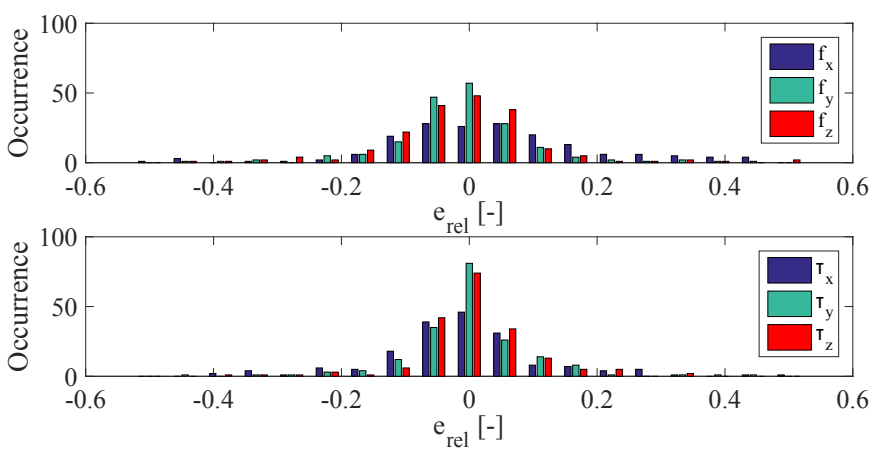

Fig. 8. Histogram of $e_{r e l}$ computed with 200 testing poses of each wrench component using experimental data of D-optimal design.

mainly distributed in $[-0.1,0.1]$, whereas the relative error of $f_{x}$ spreads more widely.

Similar to the D-optimal case, the data points with relative error beyond the interval $[-0.5,0.5]$ are located from $-5 \mathrm{~N}$ to $5 \mathrm{~N}$ for $f_{y}$ and $f_{z}$ with absolute errors lie within [-1.5 $\mathrm{N}$, $1.5 \mathrm{~N}]$, while the points of $f_{x}$ have larger absolute errors. The points of torques, whose relative error beyond $[-0.5,0.5]$, are distributed between [-0.5 Nm, $0.5 \mathrm{Nm}]$. Analogously, we solve the ill-conditioned problem with PCR with $b=0.1$. The results are shown in Table III.

In summary, the poses of both design methods yield polynomial models with similar accuracy which leads to the conclusion that the poses of the experiments do not yield an ill-posed problem and the identified models represent a good fit of the stiffness characteristics of the ECM. 

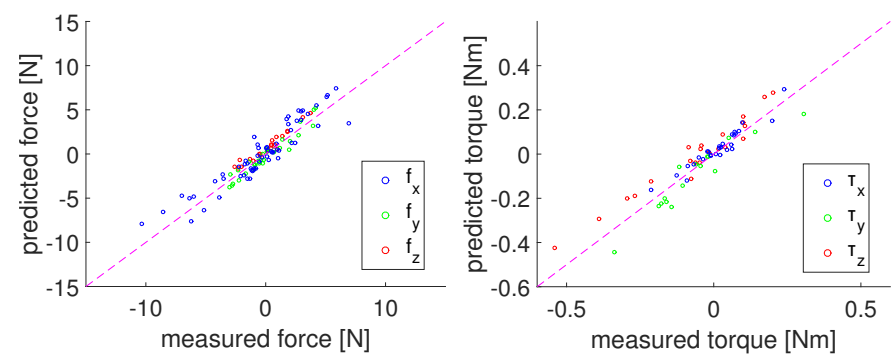

Fig. 9. Actual vs Predicted plot using data of the D-optimal design. The dashed line represents an exact prediction and is added for an intuitive comparison of the prediction results.
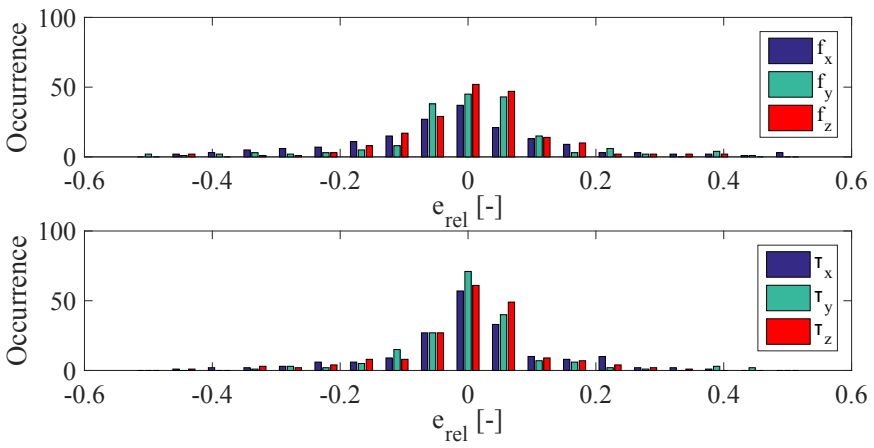

Fig. 10. Histogram of $e_{r e l}$ computed with 200 testing poses of each wrench component using experimental data of maximin-distance design.

\section{E. Computational Efficiency}

As stated in the motivation, a primary goal of the identified mapping is to be computationally efficient for the use in real time control. To investigate the computational efficiency, 100 pose-wrench pairs are randomly selected from $\mathcal{C}$, see Section II-C, and are used as input of the polynomial model and the FEM model, respectively. The required computational time of each model is measured and Fig. 11 depicts the histogram of the computation time, which states that the polynomial model is at least 200 times faster than the FEM model.

\section{F. Cartesian Stiffness Matrix}

The Cartesian stiffness matrix of the ECM can be derived analytically from the identified polynomial models by the partial derivative $\boldsymbol{K}=\partial \boldsymbol{h} / \partial \boldsymbol{p} \in \mathbb{R}^{6 \times 6}$. Considering that $\boldsymbol{K}$ is based on the measurement data, the matrix might not be perfectly symmetric because of the measurement uncertainty or anisotropic effects of the material. A symmetric Cartesian stiffness matrix can be derived from the relationship

$$
\boldsymbol{K}=\frac{1}{2}\left(\boldsymbol{K}+\boldsymbol{K}^{T}\right)+\frac{1}{2}\left(\boldsymbol{K}-\boldsymbol{K}^{T}\right)=\boldsymbol{K}_{\text {sym }}+\boldsymbol{K}_{\text {skew }}
$$

Specifying a certain pose, the corresponding symmetric and skew-symmetric sub matrix can be obtained. To check whether the symmetric matrix is dominant, the ratio of $\left\|\boldsymbol{K}_{\text {sym }}\right\|_{2}$ to $\|\boldsymbol{K}\|_{2}$ can be taken and Fig. 12 displays the histogram of this ratio of each pose measured in the experiments. It shows that all of them are greater than 0.95 , indicating the dominance of the symmetric matrix.

As an example, the symmetric Cartesian stiffness matrix of the initial configuration is calculated to

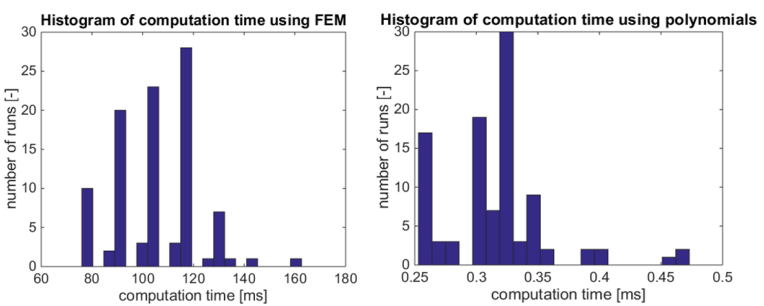

Fig. 11. Histograms of the computational time used by the FEM model (left) and the polynomial model (right) computed with 100 randomly selected poses from $\mathcal{C}$.

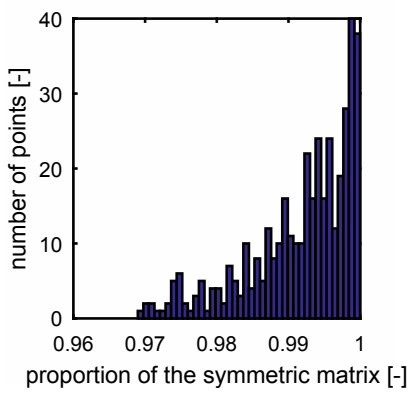

Fig. 12. Histogram of the proportion of the symmetric matrix computed with 300 testing poses from the experiment.

$$
\left.\boldsymbol{K}_{\text {sym }} \boldsymbol{p}_{\text {init }}\right)=\left(\begin{array}{cccccc}
24585 & -778 & 12 & 4 & 49 & -14 \\
-778 & 3461 & 106 & -1 & 28 & 179 \\
12 & 106 & 3468 & 0 & -178 & 43 \\
4 & -1 & 0 & 4 & 0 & 0 \\
49 & 28 & -178 & 0 & 16 & -1 \\
-14 & 179 & 43 & 0 & -1 & 16
\end{array}\right)
$$

As expected, the diagonal elements $\frac{\partial f_{y}}{\partial y}=3461 \mathrm{~N} / \mathrm{m}$ and $\frac{\partial f_{z}}{\partial z}=3468 \mathrm{~N} / \mathrm{m}$ are almost equal because of the symmetric structure of the ECM. Analogously, $\frac{\partial \tau_{y}}{\partial \theta}=16 \mathrm{Nm} / \mathrm{rad}$ and $\frac{\partial \tau_{z}}{\partial \psi}=16 \mathrm{Nm} / \mathrm{rad}$ are also equal. Compared to the $\frac{\partial f_{y}}{\partial y}$ and $\frac{\partial f_{z}}{\partial z}$, the diagonal element $\frac{\partial f_{z}}{\partial z}=24585 \mathrm{~N} / \mathrm{m}$ is significantly greater, indicating a larger translational stiffness along the $x$ direction (compression). However, the fourth element $\left(\frac{\partial \tau_{x}}{\partial \phi}=4 \mathrm{Nm} / \mathrm{rad}\right)$ is smaller in comparison with the fifth and sixth diagonal elements, which implies that twisting of the ECM is easier compared to bending in the initial configuration. The offdiagonal elements $\left(\frac{\partial \tau_{z}}{\partial y}=179 \mathrm{~N}\right.$ and $\left.\frac{\partial \tau_{y}}{\partial z}=-178 \mathrm{~N}\right)$ are significant, indicating a strong coupling between the translational displacement and the torque around the axis perpendicular to the displacement direction. This seems physically reasonable as e.g. for a planar bending of a beam-like structure, the applied torque induces a translational shear motion.

\section{CONCLUSION AND FUture WORK}

In this work, a novel experimental method is introduced that identifies the mapping from a Cartesian pose to a Cartesian wrench of an ECM. The mapping is set up by using cubic multivariate polynomials in a workspace that is larger than the reachable space by the actuation system to account for configurations which are induced by external disturbances. The basic idea of the method is to deflect the ECM to different static configuration and measure pose-wrench data pairs at 
each configuration. Three consecutive steps are presented to perform the identification. In the first step, a large data set of training poses is generated using a FEM-model that displays the deformation of the ECM. In the second step, the experimental design is carried out which essentially reduces the large data set of training poses to a minimum set that needs to be sampled experimentally. In the third step, a robot manipulator is used to automatically drive the ECM to the poses of the minimum set whereas unwanted configurations of the ECM are avoided by a control strategy using an impedance control and a position control scheme. The experimental results of the mapping are evaluated by a hold-out validation that yields a maximum root mean square error of $3.8 \mathrm{~N}$ in the forces and $0.27 \mathrm{Nm}$ in the torques whereas the polynomial model with an average computation time of $0.3 \mathrm{~ms}$ was tested to be at least 200 times faster compared with the FEM-model tested on the same computer.

As stated in the introduction, the proposed method is only applicable for mechanisms that are actuated by a rigid platform at their tip end. To extend the method to e.g. pneumatically driven mechanisms, the actuation system needs to be incorporated in the experiment. For this extension, an attached robot could be used that follow the mechanism to a desired pose and perturb it subsequently. However, the number of input parameters will change and thus a polynomial model might not be suitable.

Other topics for future works are to employ statistical measures in the decision-making process for the number of experimentally sampled poses in Sec. III or the fitting performance of an investigated model in Sec. II-C. Moreover, other types of model-free mappings could be used and tested in step 2 such as neural networks or Gaussian process regression. In these cases, the experimental design should be carried out by the model-free approach, see Sec. III-B, as an information matrix can not be computed.

\section{REFERENCES}

[1] Jens Reinecke, Bastian Deutschmann, and David Fehrenbach. A structurally flexible humanoid spine based on a tendon-driven elastic continuum. In Robotics and Automation (ICRA), IEEE International Conference on, pages 4714-4721. IEEE, 2016.

[2] Lael U Odhner, Leif P Jentoft, Mark R Claffee, Nicholas Corson, Yaroslav Tenzer, Raymond R Ma, Martin Buehler, Robert Kohout, Robert D Howe, and Aaron M Dollar. A compliant, underactuated hand for robust manipulation. The International Journal of Robotics Research, 33(5):736-752, 2014.

[3] Jennifer C Case, Edward L White, Vytas SunSpiral, and Rebecca Kramer-Bottiglio. Reducing actuator requirements in continuum robots through optimized cable routing. Soft Robotics, 2017.

[4] Bastian Deutschmann, Alexander Dietrich, and Christian Ott. Position control of an underactuated continuum mechanism using a reduced nonlinear model. In Decission and Control (CDC), IEEE International Conference on. IEEE, 2017.

[5] Bastian Deutschmann, Christian Ott, Concepcion A Monje, and Carlos Balaguer. Robust motion control of a soft robotic system using fractional order control. In International Conference on Robotics in Alpe-Adria Danube Region, pages 147-155, 2017.

[6] Maxime Chalon, Werner Friedl, Jens Reinecke, Thomas Wimboeck, and Alin Albu-Schaeffer. Impedance control of a non-linearly coupled tendon driven thumb. In Intelligent Robots and Systems (IROS), IEEE/RSJ International Conference on, pages 4215-4221, 2011.

[7] D Caleb Rucker and Robert J. Webster III. Statics and dynamics of continuum robots with general tendon routing and external loading. IEEE Transactions on Robotics, 27(6):1024-1030, 2011.
[8] Federico Renda, Michele Giorelli, Marcello Calisti, Matteo Cianchetti, and Cecilia Laschi. Dynamic model of a multibending soft robot arm driven by cables. IEEE Transactions on Robotics, 30(5):1109-1122, 2014.

[9] Jérémie Allard, Stéphane Cotin, François Faure, Pierre-Jean Bensoussan, François Poyer, Christian Duriez, Hervé Delingette, and Laurent Grisoni. Sofa-an open source framework for medical simulation. In MMVR 15Medicine Meets Virtual Reality, volume 125, pages 13-18. IOP Press, 2007.

[10] Christian Duriez. Control of elastic soft robots based on real-time finite element method. In Robotics and Automation (ICRA), 2013 IEEE International Conference on, pages 3982-3987. IEEE, 2013.

[11] Ian A Gravagne, Christopher D Rahn, and Ian D Walker. Large deflection dynamics and control for planar continuum robots. IEEE/ASME transactions on mechatronics, 8(2):299-307, 2003.

[12] Valentin Falkenhahn, Tobias Mahl, Alexander Hildebrandt, Rüdiger Neumann, and Oliver Sawodny. Dynamic modeling of bellows-actuated continuum robots using the euler-lagrange formalism. IEEE Transactions on Robotics, 31(6):1483-1496, 2015.

[13] Michele Giorelli, Federico Renda, Marcello Calisti, Andrea Arienti, Gabriele Ferri, and Cecilia Laschi. Neural Network and Jacobian Method for Solving the Inverse Statics of a Cable-Driven Soft Arm With Nonconstant Curvature. IEEE Transactions on Robotics, 31(4):823-834, 2015.

[14] A. Melingui, R. Merzouki, J. B. Mbede, C. Escande, and N. Benoudjit. Neural networks based approach for inverse kinematic modeling of a compact bionic handling assistant trunk. In IEEE 23rd International Symposium on Industrial Electronics (ISIE), pages 1239-1244, June 2014.

[15] Milad Malekzadeh, Jeffrey Queißer, and Jochen J Steil. Learning the end-effector pose from demonstration for the bionic handling assistant robot. In Proceedings of the International Workshop on Human Human Friendly Robotics, 2016.

[16] Simon Raphael Eugster. On the foundations of continuum mechanics and its application to beam theories. PhD thesis, ETH Zurich, 2014.

[17] David A Belsley, Edwin Kuh, and Roy E Welsch. Regression diagnostics: Identifying influential data and sources of collinearity, volume 571. John Wiley \& Sons, 2005.

[18] Modechai Shacham and Neima Brauner. Minimizing the effects of collinearity in polynomial regression. Industrial \& engineering chemistry research, 36(10):4405-4412, 1997.

[19] John Neter, Michael H Kutner, Christopher J Nachtsheim, and William Wasserman. Applied linear statistical models, volume 4. Irwin Chicago, 1996.

[20] Jerome Friedman, Trevor Hastie, and Robert Tibshirani. The elements of statistical learning, volume 1. Springer series in statistics Springer, Berlin, 2001.

[21] John Mandel. Use of the singular value decomposition in regression analysis. The American Statistician, 36(1):15-24, 1982.

[22] Friedrich Pukelsheim. Optimal design of experiments. SIAM, 2006.

[23] Yu Sun and John M Hollerbach. Observability index selection for robot calibration. In Robotics and Automation, 2008. ICRA 2008. IEEE International Conference on, pages 831-836. IEEE, 2008.

[24] Yong B Lim. D-optimal design for cubic polynomial regression on the q-simplex. Journal of statistical planning and inference, 25(2):141-152, 1990.

[25] Norbert Gaffke and Berthold Heiligers. Optimal and robust invariant designs for cubic multiple regression. Metrika, 42(1):29-48, 1995.

[26] Nam-Ky Nguyen and Alan J Miller. A review of some exchange algorithms for constructing discrete d-optimal designs. Computational Statistics \& Data Analysis, 14(4):489-498, 1992.

[27] Mark E Johnson, Leslie M Moore, and Donald Ylvisaker. Minimax and maximin distance designs. Journal of statistical planning and inference, 26(2):131-148, 1990.

[28] E Marengo and $\mathrm{R}$ Todeschini. A new algorithm for optimal, distancebased experimental design. Chemometrics and Intelligent Laboratory Systems, 16(1):37-44, 1992.

[29] k610 CMM Metris. http://www.metris3d.hu, 2016.

[30] Richard M Murray, Zexiang Li, and S Shankar Sastry. A mathematical introduction to robotic manipulation. CRC press, 1994.

[31] A Albu-Schäffer, S Haddadin, Ch Ott, A Stemmer, T Wimböck, and G Hirzinger. The dlr lightweight robot: design and control concepts for robots in human environments. Industrial Robot: An International Journal, 34(5):376-385, 2007. 